\section{Affect: concept + context. Architectural practice as relational space}

PALABRAS CLAVE • TEORÍA DE LA ARPUITECTURA · AFECTO CONCEPTO - CONTEXTO · ARPUITECTURAS COLECTIVAS

KEYWORDS • ARCHITECTURAL THEORY · AFFECT · CONCEPT CONTEXT. COULCTIVE ARCHITECTURES

\section{RESUMEN}

En su artículo "Concepto, contexto, contenido", Bernard Tschumi, plantea una aproximación a la práctica arquitectónica a partir del análisis de tres de sus instancias fundamentales: el concepto, el contexto y el contenido. La pregunta por la legitimidad de la prevalencia de una noción u otra inicia una reflexión en torno a la confrontación concepto-contexto trasladada al panorama arquitectónico contemporáneo. Para ello, se propone deshacer el binomio concepto-contexto mediante la introducción de un tercer término en la relación: el afecto. Esta introducción pretende abrir un camino para pensar nuevos enfoques de la práctica arquitectónica que respondan a las demandas del contexto actual.

\section{ABSTRACT}

The article "Concept, Context, Content" written by Bernard Tschumi proposes an approach to the architectural practice based on the analysis of three fundamental architectural components: concept, context and content. The question of the legitimacy of the prevalence of one or other notion pece to consider to the confrontatio text in the contemporary architectural scene. In order to explore this confrontation, the introduction of a third term in the relation is proposed: "affect", thus, allowing the binomial concept-context fade away and permitting the emergence of new approaches. The following paper claims that an affective approach to architecture can generate more accurate responses to current challenges.

\title{
Afecto: concepto + contexto. La práctica arquitectónica como espacio relacional
}

\author{
MÓNICA AUBÁN BORRELL* · Universidad Politécnica de Cataluña, España · monica.auban@upc.edu
}

Fecha de recepción: 13 febrero 2017 - Fecha de aceptación: 17 abril 2017

"El arte y la filosofía seleccionan el caos, y se enfrentan a él pero no se trata del mismo plano de sección, ni de la misma manera de poblarlo, constelaciones de universo o afectos y perceptos en el primer caso, complexiones de inmanencia o conceptos en el segundo. No es que el arte piense menos que la filosofía, sino que piensa por afectos y perceptos" (Deleuze \& Guattari, 2013).

La invitación a participar en el número 32 de esta revista hacía referencia al artículo "Concept, Context, Content" escrito por el arquitecto suizo Bernard Tschumi y publicado en Event-Cities 3 en el año 2004. Esta referencia situaba, de manera discursiva, la discusión en torno a la problemática derivada de la confrontación concepto-contexto. La lectura de dicho artículo suscitó diferentes reflexiones que sugerían una oportunidad para pensar - o repensar- posibles actualizaciones de la discusión de esta problemática en el panorama arquitectónico contemporáneo.

Cabe aclarar que la reflexión que se va a desarrollar aquí tiene, metodológicamente, un origen que es marcadamente textual. Esto quiere decir que el artículo de Tschumi va a ser entendido, en sí mismo, como una materia prima para la reflexión; como un marco autónomo en el que situarnos para poder empezar el ejercicio de reformulación. La aproximación textual debe alejarse, por tanto, de cualquier interpretación que pudiera confundir la lectura que se propone con una valoración crítica de la obra de este arquitecto. Y es que, la obra tanto construida como teórica de Bernard Tschumi cuenta ya con un recorrido tal que su valoración crítica requeriría de un análisis monográfico y riguroso, muy alejado de los objetivos que este artículo se propone.

La exploración en torno a la relación conceptocontexto-contenido por parte de Bernard Tschumi y, también, la manera en que esta relación se hace explícita en su formalización escrita, se presentan entonces como un punto de partida desde el cual comenzar a establecer conexiones entre las dimensiones teórica, práctica y contextual de la disciplina arquitectónica que el presente artículo sugiere.

Para ello, se propone establecer un diálogo entre este texto de Tschumi, desde su perspectiva marcadamente arquitectónica y un texto de

* Arquitecta (2013) por la Universitat Politècnica de València y Máster en Teoría e Historia de la Arquitectura (2015) por la Universitat Politècnica de Catalunya. Actualmente cursa estudios de doctorado en el Programa de Teoría e Historia de la Arquitectura de la Escuela Técnica Superior de Arquitectura de Barcelona (ETSAB) de la Universitat Politècnica de Catalunya. Miembro del grupo de investigación ACC-Arquitectura, Ciutat i Cultura y beneficiaria de la Ayuda Predoctoral Fi-Agaur. 
cariz propiamente filosófico como es ¿̇Qué es la filosofía? escrito por los pensadores Gilles Deleuze y Félix Guattari; un texto que también coloca a la discusión en torno al concepto en el eje vertebrador de su discurso. Considero que la confrontación de la idea arquitectónica de concepto con otras disciplinas puede ayudar a abrir el horizonte referencial en el que los arquitectos estamos habituados a movernos, afrontando así unos desafíos que, desde otras coordenadas, son capaces de interpelar y sacudir la actividad proyectual.

\section{MÁS ALLÁ DE LA OPOSICIÓN BINARIA: CONCEPTO Y CONTEXTO}

El texto "Concepto, Contexto, Contenido" de Bernard Tschumi (2005) ${ }^{[1]}$ plantea una reflexión en torno a tres instancias constitutivas del quehacer arquitectónico: el concepto, como el proceso de abstracción que orienta la definición formal de lo proyectado; el contexto, que se refiere, principalmente, a la relación de lo construido con los condicionantes del lugar de su inscripción; y el contenido, entendido este último, desde un punto de vista más funcional, como respuesta a las necesidades programáticas de la obra que se diseña.

El artículo se inicia a partir de una sentencia tajante: "no hay arquitectura sin concepto" (Tschumi, 2005, p. 78), puesto que no es posible concebir la arquitectura sin una idea general que articule sus procesos. En el mismo párrafo introductorio, Tschumi nos dice también que "no hay arquitectura sin contexto" (2005, p. 78), ya que tampoco podemos concebir una arquitectura que no se encuentre situada en un lugar concreto. Dejando a un lado el contenido -pues este parece ocupar una posición menos relevante en la argumentación de Tschumi-, nos encontramos con el concepto y el contexto como dos componentes intrínsecos y fundamentales de la arquitectura.

Ambos términos, concepto y contexto, se presentan como dos vocablos de uso común en el seno de la disciplina arquitectónica por lo que cuentan, cada uno de ellos, con unos significados asociados ampliamente extendidos. Así, tendemos a identificar el concepto con aproximaciones al ejercicio proyectual en las que prima un fuerte contenido formal que se impone de manera apriorística. Mientras que una arquitectura vinculada al contexto está comúnmente asociada a un tipo de intervenciones que encuentran en la lectura de las complejidades de cada entorno concreto el motor para su acción.

Concepto y contexto, como componentes intrínsecos, conviven en el interior del apartado arquitectónico lo que implica, necesariamente, que entre ellos ha de establecerse algún tipo de relación. En este sentido, Tschumi señala cómo pese a tratarse de dos entidades inseparables, en numerosas ocasiones, concepto y contexto entran en disputa:

“Dentro de la arquitectura, el concepto y el contexto son inseparables. Frecuentemente, también están en conflicto. El concepto puede negar o ignorar las circunstancias que lo rodean, mientras que el contexto puede oscurecer o difuminar la precisión de una idea arquitectónica" (Tschumi, 2005, p. 78).

Ante esta situación de convivencia, a veces problemática, Tschumi lanza la pregunta de si sería legítimo defender la prevalencia de una de estas instancias sobre la otra. Reconociendo que históricamente la arquitectura ha visto enfrentada las posiciones de defensa del concepto y el contexto, Bernard Tschumi parece alejarse de esta disputa situando el interés de la misma en la propia relación establecida entre ambas instancias constitutivas. Sin embargo, y pese a esta declaración de intenciones la idea de conflicto va a estar muy presente en el discurso.

En este sentido, observamos que el cuerpo principal de la reflexión está articulado en tres bloques en los que las tres instancias arquitectónicas que aparecían en el título van oponiéndose de manera consecutiva: Concepto versus Contexto(s), Concepto versus Contenido y Contenido versus Contexto(s) (Tschumi, 2005, pp. 78-82). Evidentemente, esta oposición no implica una anulación total del diálogo entre las diferentes instancias, pero sin embargo sí que determina un tipo de relación muy particular: una relación de confrontación en la que casi necesariamente alguna de las componentes tenderá a imponerse sobre la otra.

La utilización del vocablo latino versus para introducir estas relaciones resulta muy esclarecedora. Y es que, según la definición que aparece en el diccionario: [versus] indica oposición o enfrentamiento tanto material como inmaterial; se sitúa entre dos sustantivos o sintagmas sustantivos ${ }^{[2]}$. Nos encontramos así con que la exploración de las posibles relaciones entre concepto, contexto y contenido va a estar regida por la contraposición de términos; una contraposición que opera como un sistema de oposición binaria, dirigiendo los significados extraídos.

Es cierto que, en el campo arquitectónico, una confrontación bilateral del tipo concepto versus contexto puede llegar a desatar una tensión que podría ser convertida en la corriente generativa que sostuviera al propio proyecto. Pero este artículo entiende que la limitación de las relaciones entre concepto y contexto a una mera oposición binaria supone una reducción de las

\footnotetext{
[1] Aunque el texto, en su versión original fue publicado en 2004, nos remitiremos para este artículo a la versión española que apareció en el Nº 34 de la revista Arquine en el año 2005.

[2] Esta definición ha sido extraída del Diccionario Oxford en español en su versión web, disponible en https://es.oxforddictionaries.com/definicion/versus
} 
potencialidades que pueden ser asociadas a la práctica arquitectónica.

Cabe señalar que los sistemas de oposición binaria han sido ampliamente estudiados como mecanismos de confrontación en los que un término prevalece siempre sobre el otro, imponiendo su dominio y coartando las posibilidades de desarrollo del término que se ve relegado ${ }^{[3]}$. De la misma manera que Bernard Tschumi se preguntaba por la relación entre concepto y contexto, nosotros nos preguntamos si este sistema de oposición binaria es la única forma de relación que existe entre estas instancias constitutivas. $\mathrm{O}$, si por el contrario, somos capaces de pensar en un tipo de relación, más recíproca, en la que concepto y contexto se entremezclen complementándose en lugar de anulándose.

\section{LA CRISIS ECONÓMICA Y EL GIRO SOCIAL DE LA ARQUITECTURA}

Afrontar esta pregunta a la que Bernard Tschumi nos invita no constituye solo un ejercicio teórico. La tensión que históricamente se ha ido desatando entre estos dos polos de la arquitectura convierte a la propia relación concepto-contexto en un buen medidor del pulso de la arquitectura de cada época.

Aparece, en este sentido, una distancia temporal que instaura una diferencia profunda entre el panorama arquitectónico en el momento de publicación del texto de Tschumi y nuestro momento presente. Aunque, a primera vista, la escasa década que separa ambos momentos pueda parecer irrelevante, el estallido de la crisis económica en el año 2007 estableció una fractura importantísima en la definición del ejercicio arquitectónico; una fractura que tuvo también reflejo en la distribución de fuerzas entre los términos de la relación concepto-contexto ${ }^{[4]}$.

Las décadas anteriores al 2007 estuvieron marcadas, en gran medida, por un giro fuertemente mercantilista en el interior de la arquitectura que amenazaba seriamente con convertir a parte importante de esta práctica en una mera producción objetual. La emergencia del llamado star-system arquitectónico ejemplifica la consolidación de un movimiento que desde el final de los años 60 venía fraguándose; un movimiento determinado por las tensiones derivadas de la entrada de la arquitectura en los sistemas de la sociedad de consumo (Montaner \& Muxí, 201 1, pp. 42-53).

La orientación empresarial de la arquitectura de este star-system, es decir, la producción de sus obras bajo el paraguas de las marcas o el sello de autor, así como, la despreocupación, tantas veces observada, por la realidad del lugar en la que la intervención sería realizada parecía ir desplazando el peso del proceso proyectual hacia el lado del concepto. De hecho existe entre el acervo de obras de esta corriente un sesgo común en el que los juegos geométricos, los alardes estructurales y los desafíos formales se imponen como imagen predominante.

El estallido de la burbuja económica tuvo, sin embargo, consecuencias inmediatas sobre este movimiento arquitectónico. La situación de crisis puso de manifiesto el despilfarro económico que había caracterizado a los años anteriores, poniendo en jaque el modus operandi de las grandes figuras del star-system. La falta de recursos, y quizás también una nueva conciencia, abrió paso a otro tipo de intervenciones, de escala más acotada, en los que la emergencia social ha ido ganando, poco a poco, peso como eje articulador de los proyectos.

Esta situación, en la que aún nos encontramos, supone también una alteración de la relación concepto-contexto. La aparición de la dimensión social como un horizonte válido implica, al menos para un sector de la arquitectura, omitir las cuestiones formales apriorísticas, dejando vía libre para la aparición de un tipo de intervención comprometida en los que la correcta lectura del lugar se torna una necesidad fundamental.

Resulta evidente, que esta rama de la arquitectura comprometida con lo social no nació con la crisis económica y es posible encontrar distintos arquitectos que ya antes de 2007 habían colocado la dimensión social en el foco de sus actuaciones. La preocupación por la introducción del discurso ético en la arquitectura, así como la vinculación de la acción proyectual con el compromiso social han estado presentes, con mayor o menos protagonismo, en la disciplina desde finales del siglo XIX (Montaner \& Muxí, 201 1, pp. 42-53).

Remitiendo de nuevo al contexto español, un ejemplo elocuente de esta arquitectura combativa que viniera realizándose ya en el contexto precrisis lo constituyen las intervenciones del arquitecto sevillano Santiago Cirugeda, a través de la plataforma "Recetas Urbanas".

Desde 1995, Cirugeda y sus diferentes colaboradores, han venido desarrollando una acción arquitectónica subversiva basada, principalmente, en una crítica tanto al sistema de consumo como al sistema institucional

[3] Una crítica muy fructífera a los sistemas de oposición binaria es la que ha sido desarrollada por la rama del feminismo que defiende el enfoque de la llamada diferencia sexual. El trabajo de pensadoras contemporáneas como Rosi Braidotti (2005) consigue combinar esta crítica al binarismo mediante aproximaciones a la noción de afecto que van a ser fundamentales para este artículo.

[4] Aunque el estallido de la crisis del 2007 tuvo una extensión prácticamente mundial, centraremos el análisis de este artículo al caso concreto español. La profunda vinculación entre el crecimiento económico de España y su parque inmobiliario, trajo consigo unas consecuencias devastadoras para el sector de la construcción en conjunto que, prácticamente, vio paralizado su actividad, tras varios años de actividad muy intensa. 
1. Huerto urbano en un solar vacante. Poble Nou, Barcelona. Fotografía de la autora, julio de 2014.
2. Asamblea en el encuentro de Arquitecturas Colectivas. Espai Germanetes, Barcelona. Fotografía de la autora, julio de 2014.
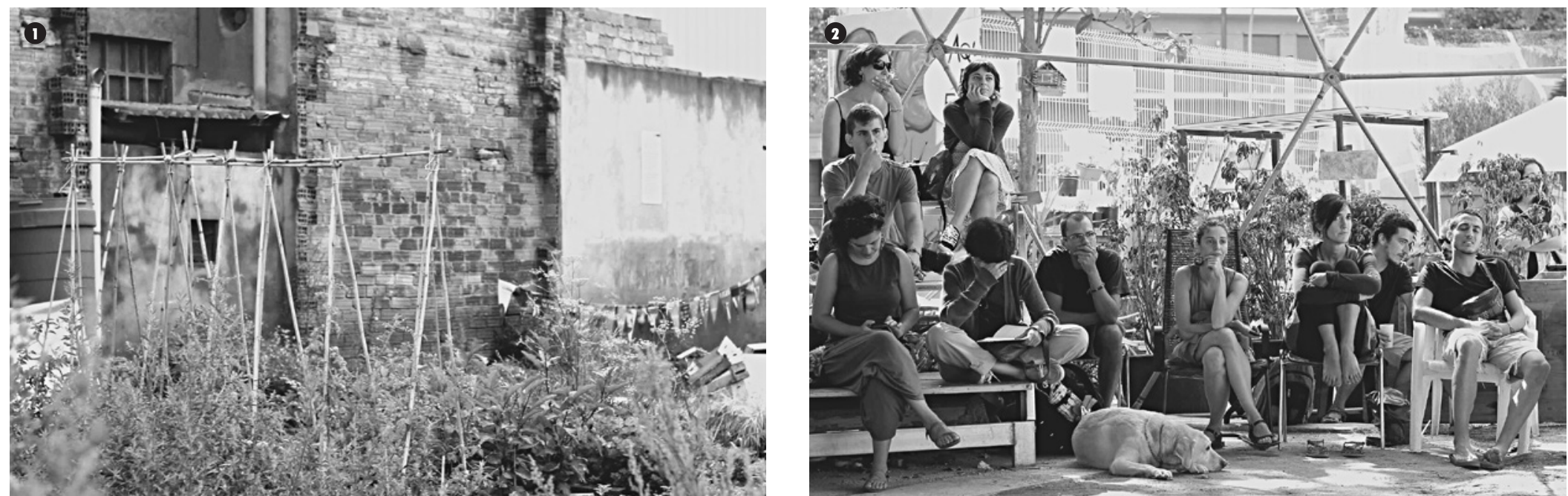

que dictan los ritmos del cambio de la ciudad contemporánea. En este sentido, las recetas urbanas recogen un amplio abanico de soluciones arquitectónicas que tratan de implicar a la ciudadanía en el diseño de su entorno inmediato, generando situaciones en las que la tensión entre legalidad e ilegalidad son llevadas a su límite máximo (Cirugeda, 2007).

La efectividad de las soluciones propuestas depende sustancialmente de un conocimiento profundo del contexto intervenido. Este conocimiento debe tener en cuenta la participación del mayor número de los agentes que componen la acción urbana: desde los vecinos y usuarios hasta las normas y regulaciones urbanísticas vigentes.

El panorama que dejó tras de sí la crisis económica puso de manifiesto que los ataques al modelo por parte de arquitectos como Santiago Cirugeda estaban absolutamente justificados y la búsqueda de otra manera de hacer arquitectura -alejada del anquilosamiento administrativo e institucional- no solo era posible, sino urgente. Así, hemos asistido en los últimos años a una proliferación de los llamados colectivos de arquitectura, equipos principalmente jóvenes que están alterando la concepción del tradicional estudio de arquitectura en una exploración de soluciones alternativas, capaces de adecuarse mejor a las demandas concretas del contexto ${ }^{[5]}$.

Nuestro análisis de la relación conceptocontexto nos ha conducido, nuevamente, a un punto en el que la noción de contexto parece imponerse por sobre la del concepto. Resulta evidente, que la nueva manera de hacer colectiva está absolutamente ligada a unos ejercicios de lectura del lugar, inseparables de la definición de contexto que Bernard Tschumi proponía en su artículo. Una definición que nos presenta al contexto en un sentido amplio, esto es, una idea de contexto que no se limite al análisis de las variables físicas o geográficas, sino que sea capaz de incorporar también las complejidades asociadas a las componentes sociales, políticas, históricas e interpersonales que perviven en cualquier territorio habitado.
Pero, ટ̇implica necesariamente esta puesta en valor del contexto dejar a un lado la idea de concepto?

\section{CONCEPTOS, FUNCTORES, AFECTOS}

Para poder enfrentar esta nueva cuestión debemos remitirnos a otra pregunta -posiblemente anterior-; una pregunta que interrogue sobre la propia naturaleza del concepto, una pregunta que se plantee, en fin ¿qué es un concepto?

El texto de Bernard Tschumi nos presenta el concepto como "una idea general, un diagrama o un esquema que da coherencia e identidad a un edificio. El concepto, no la forma, es lo que distingue a la arquitectura de la mera construcción" (Tschumi, 2005, p. 78). La capacidad para dar coherencia e identidad que Tschumi asocia al concepto nos va a permitir conectar su idea de este término con una suerte de matriz generativa, es decir, nos va a permitir entender el concepto como una entidad creativa

[5] En este sentido, el número 145 de la revista Arquitectura Viva estuvo dedicada a la actividad de varios colectivos de arquitectura que trabajan a lo largo de la geografía española. Este número fue contestado y completado por Josep Maria Montaner (2013) en el periódico El País, por considerar que su enfoque era excesivamente centralista. Para un panorama de la actividad más reciente de estos colectivos puede consultarse: https://arquitecturascolectivas.net/ 
que posibilita la emergencia de la propia arquitectura. Si es el concepto quien dota de identidad y coherencia, podemos reafirmar entonces que esta instancia es constituyente y que participa, por tanto, en la conformación de la propia obra, haciéndola reconocible como singularidad.

La definición de concepto que hemos extraído de Tschumi se encuentra enmarcada entre los límites de la disciplina arquitectónica. Sin embargo, no podemos obviar que las ideas y la utilización de los conceptos superan ampliamente estos límites.

En 1991 los pensadores Gilles Deleuze y Félix Guattari publicaron el libro ¿̇Qué es la filosofía? ${ }^{[6]}$. En él, se recoge una visión particularísima de la historia de la filosofía; una visión en la que, precisamente la reflexión en torno a la idea de concepto sostiene el peso fundamental de la aportación de ambos pensadores. Así, ¿¿Qué es la filosofía?, explora la naturaleza y la definición del propio término concepto, pero, sobre todo, explora el uso intenso que por parte de otras disciplinas se ha hecho del término.

Según un gesto que puede resultar dogmático, Deleuze y Guattari defienden, ya desde la misma introducción, la tesis de que los conceptos constituyen una pertenencia exclusiva de la filosofía, quedando el resto de disciplinas aparentemente huérfanas de esta idea. Esta defensa se inicia a partir de una crítica apoyada el estilo mordaz que caracteriza a estos autores- hacia la extensión desmedida que la noción de concepto experimentó en las últimas décadas del siglo xx. Deleuze y Guattari (2013, p. 16) nos señalan cómo desde la informática a la mercadotecnia toda la realidad parece estar regida por el régimen de los conceptos, hasta el punto de que la campaña comercial para promocionar un paquete de tallarines ha sido ya convertida en un alegato del verdadero concepto.

Evidentemente, la disciplina arquitectónica no escapa de este régimen, así vemos cómo la búsqueda de conceptos que articulen y den vida a los proyectos es una constante que se impone desde las correcciones en los talleres en las diferentes escuelas de arquitectura hasta las propuestas de participación en los más prestigiosos concursos. Pero, 己̇es este concepto que sobrevuela la arquitectura el mismo que Deleuze y Guattari reclaman en exclusiva para la filosofía? Y si respondiéramos a esta pregunta afirmativamente: żqué puede sustituir en el campo arquitectónico la idea de concepto del que ha sido desprovisto?

Los propios Deleuze y Guattari introducen un avance de la respuesta justo al inicio de su libro:

"Si la filosofía consiste en esta creación continuada de conceptos, cabe evidentemente preguntar qué es un concepto en tanto que Idea filosófica, pero también en qué consisten las demás Ideas creadoras que no son conceptos, que pertenecen a las ciencias y a las artes, que tienen su propia historia y su propio devenir, y sus propias relaciones variables entre ellas y con la filosofía" (Deleuze \& Guattari, 2013, p. 14).

Vemos, pues, que si bien los conceptos han sido expulsados del territorio de las ciencias y las artes, ambas disciplinas mantienen aún unas ideas creadoras, también ellas exclusivas y propias. Podemos afirmar entonces que la filosofía, la ciencia y las artes comparten un algo -al que ya no podremos llamar concepto- que es constitutivo y que permite dar sentido a sus diferentes actividades.

El vacío generado por la ausencia de conceptos va a ser completado por los llamados functores y afectos, capaces de sostener a las ciencias y a las artes respectivamente. Aunque no vayamos a profundizar en ellos, cabe señalar que los functores son los elementos de las proposiciones que la ciencia tiene como objeto, dentro de sus propios sistemas discursivos (Deleuze \& Guattari, 2013, pp. 117-135). Mientras que, por su parte, los afectos ${ }^{[7]}$ constituyen bloques dinámicos de sensación capaces de generar, a partir de la propia experiencia artística, intensidad y deseo (Parr, 2010, pp. 12-14).

\section{CREANDO NUEVOS MUNDOS POSIBLES}

Nos encontramos con que conceptos, functores y afectos nombran a ese algo que, es común y a la vez propio, que filosofía, ciencia y arte comparten. Para tratar de comprender en qué consiste ese nexo compartido debemos atender, en primer lugar, a las particulares connotaciones que Deleuze y Guattari están asociando a la idea de creación.

Leíamos en la cita anterior que, por un lado, la filosofía es la creación continuada de conceptos $y$, por otro lado, que las ideas que pertenecen por derecho propio a las artes y a las ciencias, es decir los functores y los afectos, son también definidas como ideas creadoras. De esta manera, y en contra de una tradición occidental de pensamiento mayoritaria que se ha centrado en el carácter contemplativo de la filosofía, la ciencia y, también, el arte; Deleuze y Guattari introducen a estas disciplinas bajo una concepción de creación

[6] La obra de Gilles Deleuze y Félix Guattari se enmarca en la corriente filosófica del postestructuralismo, una corriente de pensamiento que emergió a mediados de los años 60, principalmente en Francia, y que ha tenido importantes resonancias para la disciplina arquitectónica. Concretamente, el libro żQué es la filosofía? pertenece a un conjunto de cuatro obras que Deleuze y Guattari escribieron colaborativamente, entre los que la serie Capitalismo y esquizofrenia destaca como aportación fundamental de estos dos autores.

[7] En relación al término afecto debemos señalar la enorme repercusión que los estudios en torno a esta noción ha tenido en diferentes campos culturales. La recuperación de la figura del filósofo barroco Baruch Spinoza y su introducción en los planes de estudio de filosofía por parte de pensadores como Louis Althusser o el propio Gilles Deleuze en los años 70 supuso una revitalización de la noción de afecto. Así, hacia finales de los años 90 tendrá lugar el llamado giro afectivo en las ciencias sociales y las humanidades, un giro en los que la experiencia encarnada y corporizada será reivindicada como elemento fundamental para los análisis culturales. 
que es profundamente activa y dinámica. Crear, producir algo nuevo, provocar acontecimientos, hacer emerger... se constituyen, todas ellas, como acciones comunes a los tres campos de la filosofía, la ciencia y el arte.

Profundicemos un poco más en el giro operado por estos autores en la concepción de la actividad creadora analizando los tres aspectos principales de la acepción de creación que Deleuze y Guattari nos proponen. Estos aspectos son: el carácter productivo, el carácter activo y, por último, el carácter dinámico.

En primer lugar, Deleuze y Guattari están introduciendo una concepción de la creación en un sentido eminentemente productivo. Resulta obvio que estos autores no están, en ningún caso, tratando de defender la producción de la filosofía o el arte en términos mercantilistas o utilitarios. De hecho, a lo largo del texto insisten en reiteradas ocasiones en que la filosofía no sirve para nada, siendo precisamente esa ausencia de utilidad lo que la salva (Deleuze \& Guattari, 2013, pp. 14-15). Filosofía, ciencia y arte producen según un sentido creativo, es decir, producen alternativas y posibilidades; producen unos mundos posibles en los que nuestra propia experiencia se ve interpelada y modelada casi de manera continuada.

Evidentemente, la actividad artística siempre ha estado asociada a una dimensión creativa. Pero lo que debe llamar nuestra atención es que, en este caso, la carga de la creación o de la producción no recae sobre los productos acabados del arte, sino que recae sobre esa capacidad con la que el arte cuenta para hacer emerger situaciones en los que nuestros sistemas estables son sacudidos.

Si volvemos a situarnos sobre el campo arquitectónico vemos como esta componente productiva de la creación va a ser la que permita recuperar, más adelante, la definición de concepto como una matriz generativa que dota de identidad y coherencia que Bernard Tschumi había propuesto. La arquitectura, independientemente de su discutible inscripción o no en el campo del arte, puede encuadrarse en el marco de las actividades creadoras. Si mantenemos el sentido propuesto por Deleuze y Guattari, la arquitectura es, efectivamente, una actividad creadora en tanto que cuenta con un inestimable poder para alterar y producir esas otras realidades, al estar sus intervenciones siempre asociadas a una modificación del territorio.

Pasemos ahora a analizar las implicaciones del carácter activo asociado a la creación. Para Deleuze y Guattari los conceptos -y sus equivalentes functores y afectos- no constituyen, en modo alguno, entidades abstractas alejadas de la experiencia concreta. Todo lo contrario, cada concepto, al ser creado, responde directamente a una experiencia vivida hasta el punto de que un concepto será siempre inseparable de las circunstancias que motivaron su aparición (Parr, 2010, p. 54).

De esta manera, la actividad filosófica, la actividad científica y, por supuesto, la actividad artística implican un grado de intimidad tanto con la propia experiencia como con el entorno de aparición que convierte a conceptos, functores y afectos en entidades profundamente relacionales. En este sentido, la acción de crear está fuertemente influenciada por todo tipo de interferencias y estímulos que pueden ser tanto internos como externos (Deleuze \& Guattari, 2013, pp. 25-30). Así, un concepto o un afecto, cuando son creados, reaccionan ante estos estímulos que los envuelven generando unos nuevos sistemas o entidades que intentan dar respuesta a las condiciones concretas del medio que le interpela. El carácter relacional que es asociado a estas entidades, su capacidad para producir conexiones y encuentros entre distintos elementos, llega a ser presentado como un aspecto constitutivo e intrínseco, de manera, que encontraremos, a lo largo del texto, diferentes afirmaciones que sostienen que un concepto carecerá totalmente de sentido si se concibe de manera aislada (Deleuze \& Guattari, 2013, p. 80)
Esta componente relacional nos lleva directamente al último aspecto que proponíamos analizar: el carácter dinámico. Las ideas creadoras, tal y como son presentados por Deleuze y Guattari deben ser entendidas como unas entidades casi "vivas"; unas entidades en permanente estado de transformación cuya configuración se ve constantemente alterada por las influencias del medio circundante. Así, lejos de remitir a realidades estáticas o anquilosadas, los conceptos, los functores y los afectos ponen en circulación una idea de cambio que los define asegurando la relación con la experiencia a la que nos referíamos anteriormente.

Agrupando las tres características, las ideas creadoras de la filosofía, la ciencia y el arte pueden ser comprendidas como conjuntos de acción que nos ayudan a movernos por las diferentes experiencias que conforman una vida. Estos conjuntos de acción se nos presentan además como entidades inestables y en permanente transformación capaces, a su vez, de alterar las condiciones que consideramos estables forzándonos a afrontar nuevas situaciones.

\section{ARQUITECTURA Y AFECTO}

Tratemos de pensar ahora las implicaciones que esta noción de concepto como idea creadora pueda tener en el ámbito arquitectónico. En primer lugar, y si seguimos las indicaciones de Deleuze y Guattari, la consecuencia más inmediata ha de ser la sustitución, en nuestro discurso, de la palabra concepto por la palabra afecto, ya que la arquitectura permanece ajena al campo filosófico. Es obvio que esta sustitución derivará en otras consecuencias cuyo alcance se sitúe más allá del vocablo empleado. En este sentido, la utilización del término afecto propicia una reformulación de la noción de concepto en el campo de la arquitectura a la que Tschumi hacía referencia.

Recuperando las cualidades intrínsecas de las llamadas ideas creadoras, observamos 
3. Construcción en la playa. San Sebastian. Fotografía de la autora, marzo de 2015.

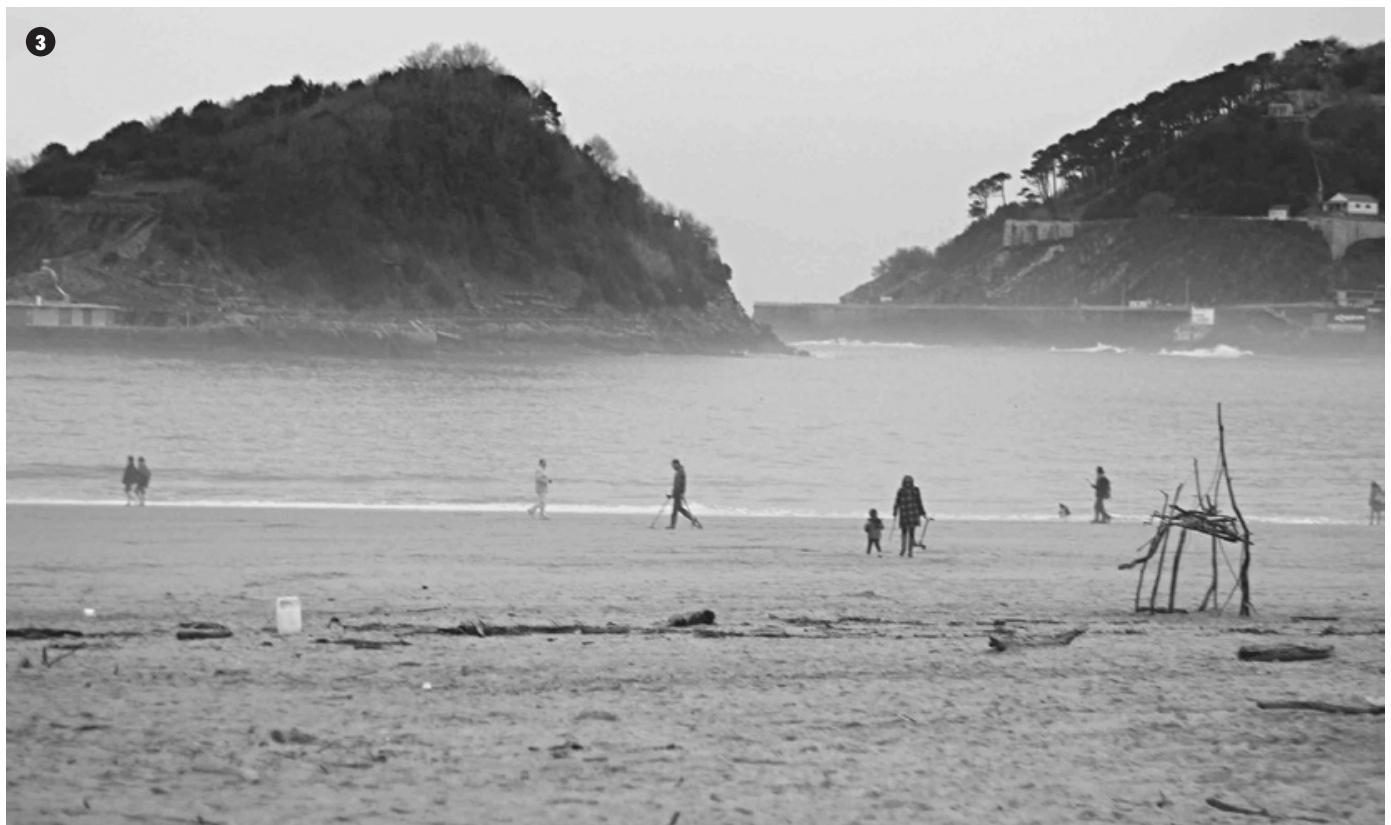

cómo la capacidad relacional es también una característica constitutiva del afecto ${ }^{[8]}$. Al situarnos en el campo arquitectónico, esta capacidad se torna un valor fundamental pues remite a un aspecto específico de la práctica proyectual: la vinculación y transformación del territorio habitado.

En el capítulo dedicado al afecto Deleuze y Guattari señalan:

"Cada territorio, cada hábitat, une sus planos o sus lienzos de pared no solo espacios-temporales, sino cualitativos: por ejemplo una postura y un canto, un canto y un color, unos perceptos y unos afectos. $Y$ cada territorio engloba o secciona territorios de otras especies, o intercepta unos trayectos de animales sin territorio, formando uniones interespecíficas" (Deleuze \& Guattari, 2013, p. 187).
Así, y mediante la introducción de pares complementarios: la tela de araña y la mosca; la caracola y el molusco; la garrapata y un mamífero indeterminado; 0 , las ya convertidas en emblemáticas por estos autores, orquídea y abeja (Deleuze \& Guattari, 2012, pp. 9-32), Deleuze y Guattari ilustran un complejo sistema de apropiaciones para la producción de territorio del que la arquitectura bien puede formar parte. De hecho, Deleuze y Guattari sitúan a la arquitectura como la primera de las artes debido al vínculo privilegiado que esta disciplina tiene con la casa pero, sobre todo, con el territorio (Deleuze \& Guattari, 2013, pp. 177-190).

Para profundizar en el encuentro entre arquitectura y afecto será fundamental entonces conocer la manera en la que estos pensadores entienden el territorio. Para Deleuze y Guattari una de las características fundamentales del territorio estriba en la capacidad del mismo para hacer emerger cualidades sensibles capaces de generar formas propias de espacialidad (Deleuze \& Guattari, 2013, p. 186). Nos encontramos pues ante una concepción territorial que no se limita a las propiedades geográficas o físicas. El canto de un pájaro es ya un territorio, y, la arquitectura, a partir del juego de relaciones que su misma implantación desata, tiene la posibilidad de participar de esta noción de territorialidad compleja.

Encontrábamos en la definición de contexto por parte de Tschumi una lectura ampliada, que nos acerca a la concepción de territorio por parte de Deleuze y Guattari. Pero, además, el peso de la noción de territorio que los pensadores franceses nos introducen descansa también sobre unas cualidades casi intangibles que no se limitan exclusivamente a cuestiones políticas, sociales o históricas. Reconocemos, en este sentido, toda una serie de delicados equilibrios que persisten en cada lugar y con los que la arquitectura, en su proceso de creación, se encuentra y enfrenta: la relación de los habitantes con su entorno, las vivencias concretas del paisaje, el sentimiento de pertenencia hacia un sitio, la identificación con la casa, con el hogar... se presentan, todas ellas, como variables profundamente afectivas -en tanto que son estas variables intangibles las que determinan la experiencia corporal en relación al entorno y al hecho construido- y, a la vez, constituyentes del ejercicio arquitectónico, ya que estas variables participan, también, en la conformación de la propia espacialidad.

Empezamos así a entrever cómo la noción de afecto nos puede permitir abordar la relación entre las nociones concepto y contexto según un sistema complementario o de reciprocidad y ya no de opuestos. Y es que el afecto, entendido como idea creadora de la arquitectura, consigue aunar bajo un solo término tanto

[8] Dentro de las diferentes aproximaciones a la teoría del afecto que pueden encontrarse actualmente, el presente artículo se encuentra vinculado a la particular interpretación de esta noción realizada por Gilles Deleuze. Esta interpretación establece una continuidad con las aportaciones de Baruch Spinoza, y está recibiendo una actualización contemporánea de la mano de la filósofa Rosi Braidotti. Así, la línea Spinoza-Deleuze-Braidotti centra su atención en la definición del afecto como capacidad para afectar y ser afectado, es decir, el afecto entendido como un mecanismo de relación complejo que nos comunica e implica con los otros y con el medio circundante. 
4. Parque urbano en el antiguo aeropuerto Tempelhof, Berlín. Fotografía de la autora, abril de 2017.

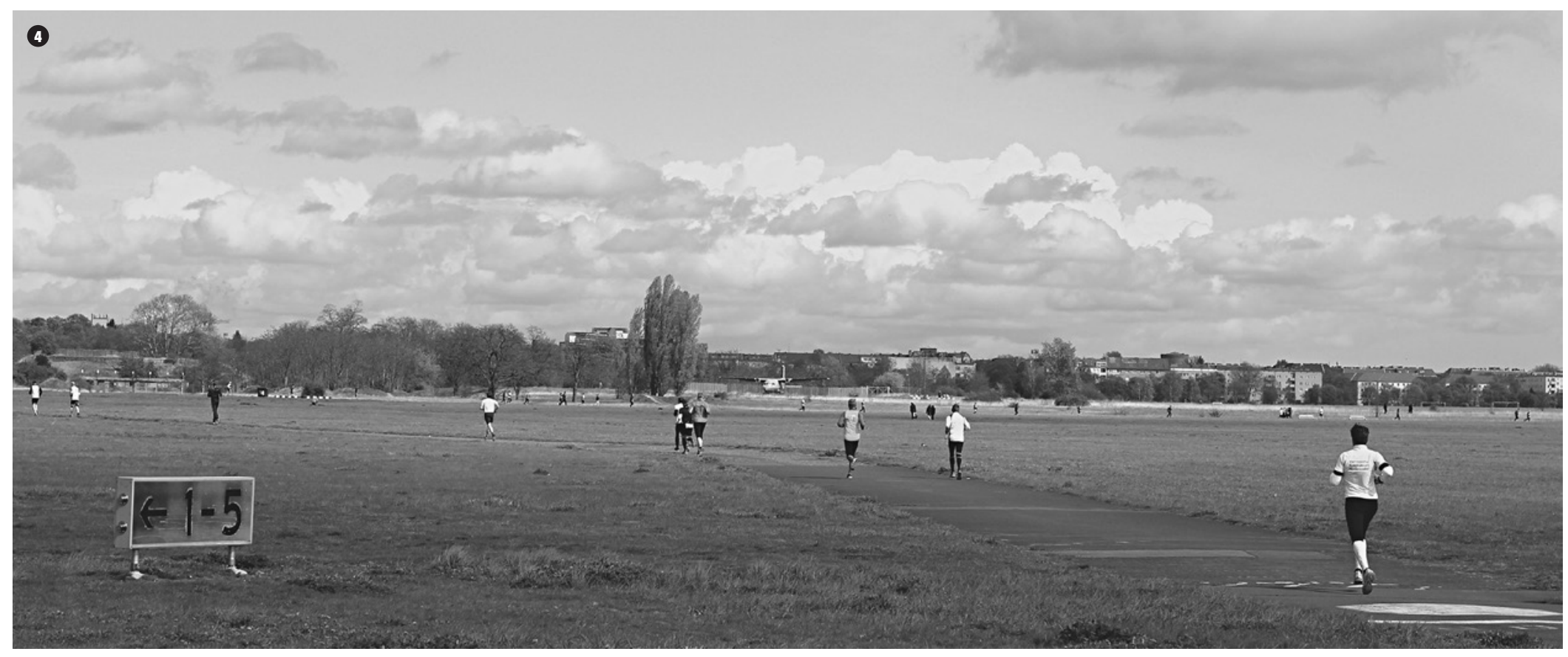

las connotaciones referidas a la matriz generativa como aquellas que se referían a las circunstancias concretas que preexisten en cada lugar que Bernard Tschumi identifica en su texto con concepto y el contexto respectivamente. La posibilidad de alcanzar este frágil equilibrio de reciprocidad entre el concepto y el contexto es vital para el ejercicio arquitectónico.

Retomemos ahora el discurso de esa arquitectura contemporánea que está poniendo en valor la dimensión social del ejercicio proyectual. Si pensamos en las aproximaciones colectivas a la práctica arquitectónica desde la perspectiva del afecto que Deleuze y Guattari nos presentan, vemos cómo la carga creativa que tradicionalmente asociamos a la idea de concepto y, que parecía haber sido relegada, aparece, de manera implícita, en los propios procesos de intervención.

La acción creadora asociada al afecto arquitectónico depende sustancialmente de unas cualidades territoriales -inmateriales e intangibles en muchas ocasiones- que emergen de las diferentes relaciones entre habitantes y entorno. En este sentido, la preocupación por realizar lecturas del lugar de intervención comprometidas con las demandas concretas que ciertas prácticas arquitectónicas realizan puede ser también entendida en términos de creación. Así, las lecturas exhaustivas de los entornos de intervención dejan de ser una referencia exclusiva a la noción de contexto pues la implicación de la acción arquitectónica con las cualidades territoriales -preexistentes y futurases ya un ejercicio creativo según el sentido expresado en ¿̇Qué es la filosofía?

\section{¿ेHACIA UNA ARQUITECTURA AFECTIVA?}

La aproximación a la arquitectura desde el afecto nos permite comprender la práctica proyectual como un espacio en disputa en el que un sinfín de relaciones cambiantes están permanentemente en juego. La mera situación de la práctica en esta perspectiva se constituye en sí misma como el carácter de identidad que convierte a cada intervención en una singularidad, con independencia del resultado formal o material obtenido.
En términos de Deleuze y Guattari, la acción creadora no se sustenta en el genio inspirado de un autor, sino que la creatividad consistirá en ser capaz de responder adecuadamente a unos estímulos concretos; a ser capaz de vincularse con las experiencias que atraviesan la realidad; a estar abiertos a asumir el cambio y a renegociar, permanentemente, las tensiones y los espacios de encuentro. El desarrollo de una práctica que sea capaz de articular las relaciones que se establecen entre diferentes actores y, también, las de estos con el entorno, se constituye como una buena manera para empezar a pensar la arquitectura desde el afecto.

En este sentido, la intervención ciudadana en el aeropuerto en desuso Tempelhof en Berlín resulta muy significativo. Tras varios años de abandono de la actividad aeroportuaria, en el año 2010 y ante la pasividad institucional, las antiguas pistas fueron ocupadas por diferentes usos e instalaciones efímeras que a día de hoy sigue funcionando como un enorme parque. El Tempelhof Feld se muestra así como una potente intervención urbana en la que la apropiación 
del lugar en desuso, y el uso intensivo por parte de los habitantes desplaza a una materialidad arquitectónica, prácticamente inexistente. Nos encontramos ante un claro ejemplo en el que el éxito del proyecto recae sobre la capacidad de lo arquitectónico para constituirse como un facilitador de relaciones y puntos de encuentro entre diferentes realidades tangibles e intangibles.

La exploración de dos de los componentes señalados por Bernard Tschumi como constitutivos de la arquitectura: el concepto y el contexto, nos ha conducido hasta la noción de afecto como un espacio intermedio en la que ambos componentes interactúan, sin excluirse, para promover una práctica creativa y capaz de dialogar con las necesidades específicas de cada entorno concreto.
Aunque las aportaciones del giro afectivo aún estén lejos de nuestra disciplina, creo que la búsqueda de este tipo de enfoque dentro de la arquitectura puede convertirse en un aporte fundamental para hacer frente a muchos los nuevos desafíos globales a los que nos enfrentamos como la crisis económica, el reto de la sostenibilidad, la informalidad como respuesta al problema de la vivienda o los procesos de gentrificación. La aproximación a la arquitectura como una práctica capaz de generar, articular y potenciar distintas relaciones, conexiones y encuentros puede ser un punto de inicio para comenzar a buscar respuestas alternativas a estos y otros desafíos contemporáneos.

\section{REFERENCIAS BIBLIOGRÁFICAS}

Braidotti, R. (2005). Metamorfosis. Hacia una teoría materialista del devenir. Madrid: Ediciones Akal.
Cirugeda, S. (2007). Situaciones urbanas. Barcelona: Editorial Tenov.

Deleuze, G. \& Guattari, F. (2012). Mil mesetas. Capitalismo y esquizofrenia. Valencia: Pre-Textos. Deleuze, G. \& Guattari, F. (2013). ¿̇Qué es la filosofía? Barcelona: Anagrama.

Montaner, J. M. \& Muxí, Z. (2011). Arquitectura y política. Ensayos para mundos alternativos. Barcelona: Editorial Gustavo Gili.

Montaner, J. M. (2013). "Colectivos de Arquitectos". El País. Recuperado de http:// ccaa.elpais.com/ccaa/2013/02/06/ catalunya/1360177819_587334.html en febrero de 2017.

Parr, A. (ed.) (2010). The Deleuze Dictionary. Edinburgh: Edinburgh University Press.

Tschumi, B. (2005). "Concepto, Contexto, Contenido". Arquine (34).

Fernández-Galiano, L. (ed.) (2012). Colectivos españoles. Nuevas formas de trabajo: redes y plataformas. Arquitectura Viva (145) 